\title{
Design and Manufacture of the Wireless Holter Monitoring Based -on ARM Micro-Controller
}

\author{
Mohammad Javad Shahrokhi' ${ }^{1}$ Hamid Reza Sadr Manouchehri Naeini*
}

${ }^{1}$ Department of Electrical Engineering, Damavand Science and Research Branch, Islamic Azad University, Damavand, Iran ${ }^{2}$ Department of Electrical Engineering, Damavand Branch, Islamic Azad University, Damavand, Iran

\section{A BSTRACT}

Introduction: Medical engineering systems nowadays play a very significant role in the diagnosis of various diseases. One of the most applicable devices of the medical engineering are receiving and recording devices of the heart signals, such as electrocardiograph. Application of Holter monitoring devices could be known as one of the substantial methods of electrocardiograph during daily activities. In this article, design and manufacture of Holter monitoring device based-on ARM micro-controller are proposed. Materials and Methods: Atmel's SAM3S Chip from the family of ARM has an eleven-channel transformer of analogue to digital which can transform analogue signals to digital values with 12 Bit accuracy by utilizing from ARM architecture used in the core of Cortex-M3 with the structure of Thumb2 processes the information with the speed of $60 \mathrm{MHZ}$. Furthermore, for receiving and reinforcement of heart signals, INA331 Chip, the product of TI Company was used. Using Chip CYRF6936, signals were transferred to the physician computer with maximum speed of one megabit/sec. Results: AT91Sam3S with the existence of the Internal Interface Circuit for communication with the external devices has enabled the system to be connected to memory cards and computer without requirement to any other interfaces. This instrumental amplifier with a single-polar feedback has $94 \mathrm{~dB}$ Common-mode rejection ratio and $54 \mathrm{~dB}$ amplification ratio. This chip has decreased the consumed power of the vehicle to $330 \mathrm{~mW}$, which by using Lithium- polymer battery of $3000 \mathrm{mAH}$, the vehicle can be fed for one day without need for charge. Conclusion: Using wireless communication, keeping the quality of the received signal from body, limitation of information reservation was removed. Furthermore, by simplifying analogue circuits, consumed power of the device was reduced and thus time used from the device was increased without the need for recharge. In addition, by using from lowconsumption 32-Bit ARM processor, high processing power and digital process capability on the heart signals will be possible easily.

\section{Key words:}

1. Wireless Technology

2. Electrocardiography 3. Low-Level Light Therapy

"Corresponding Author: Hamid Reza Sadr Manouchehri Naeini

E-mail: hr_sadr@damavandiau.ac.ir 


\title{
طراحى و ساخت هولترمانيتورينَ بىسيم بر پايةٔ ميكروكنترلر ARM
}

\author{
محمدجواد شاهرخى'، حميدرضا صدر منوجهرى نائينى r" \\ 'كروه مهندسى برق، واحد علوم و تحقيقات دماوند، دانشَاه آزاد اسلامى، دماوند، ايران

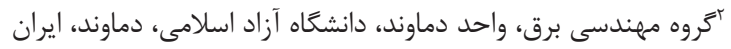

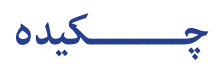

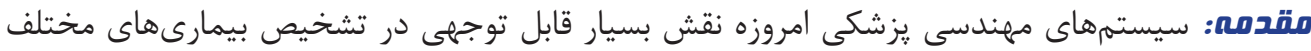

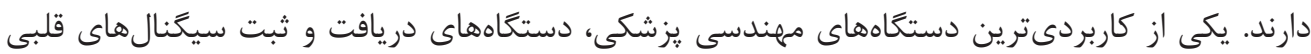

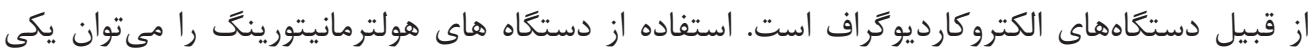

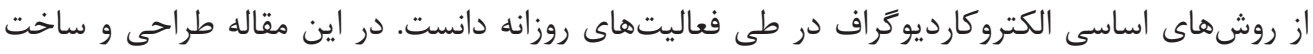

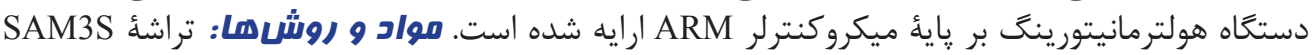

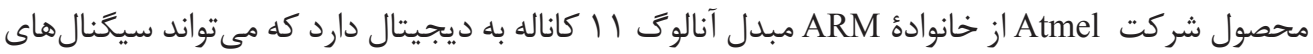

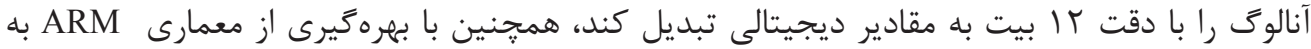
كار رفته در هستع Cortex-M3 با ساختار

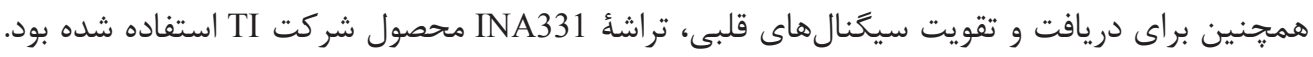

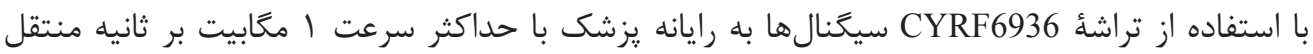

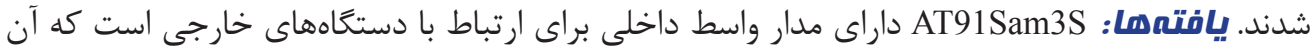

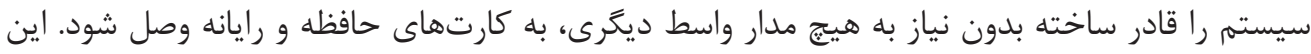

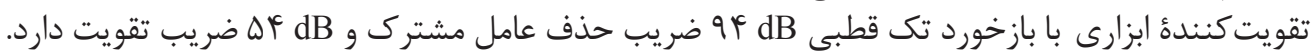

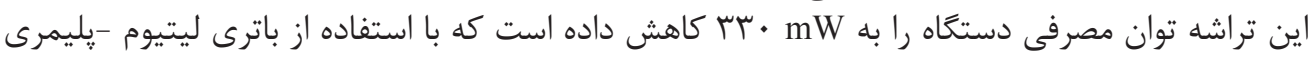

كليد وازهها:

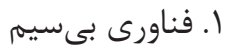

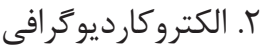
r. نور درمانى سطح يايين
إن تر ت . mAH

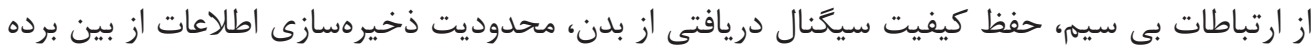

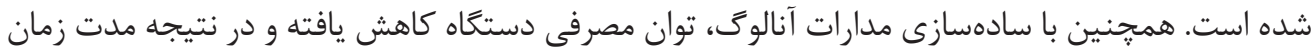

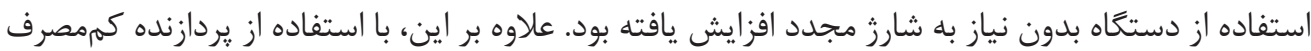

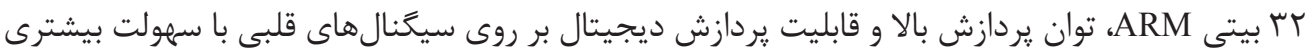
ممكن خواهد شد.

\footnotetext{
" نويسنده مسئول: حميدرضا صدر منوجهرى نائينى آدرس الكترونيكى: hr_sadr@damavandiau.ac.ir
} 


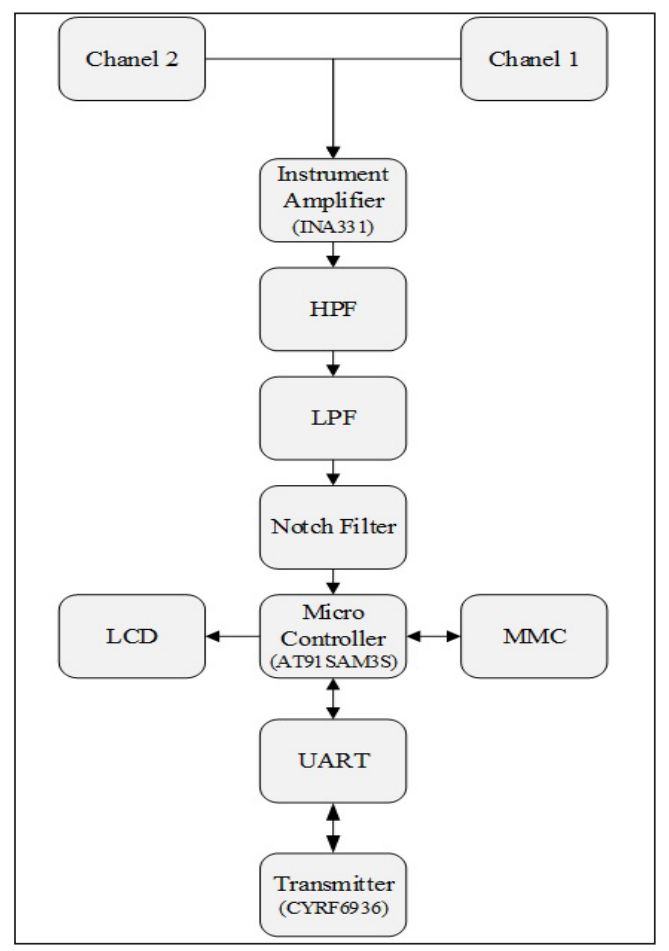

تصوير 1-بلوك دياكرام سيستم (؟).

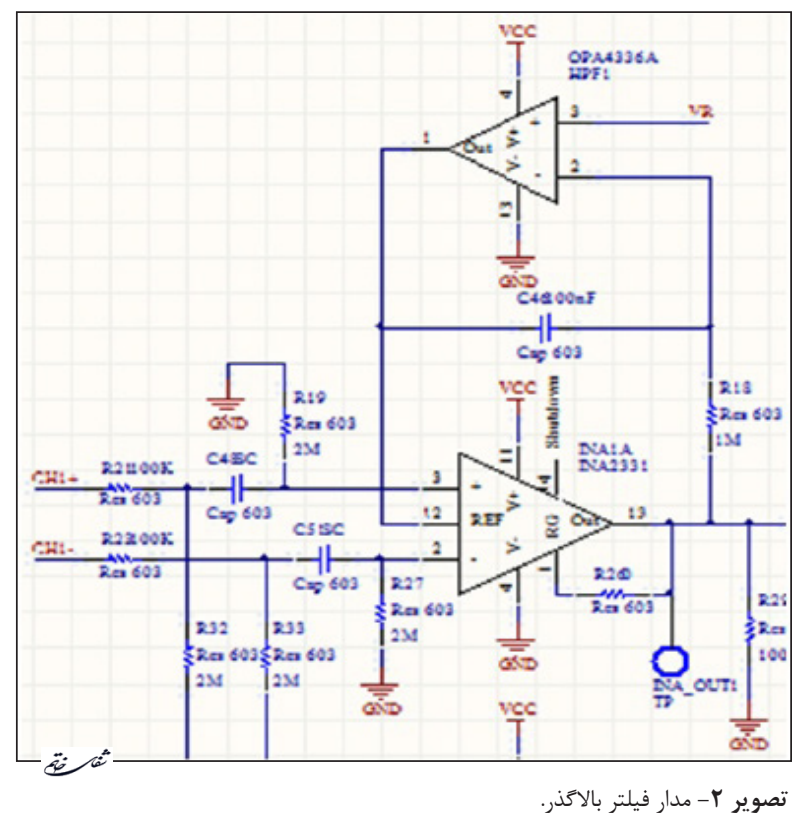

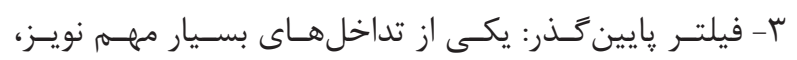

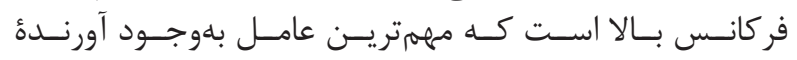

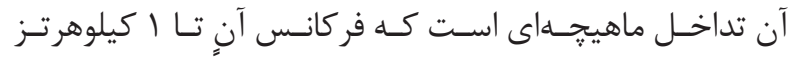

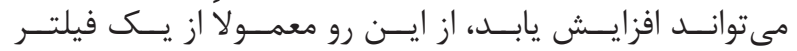

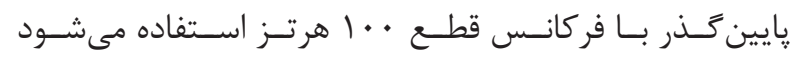

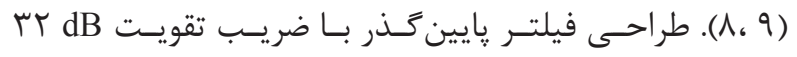

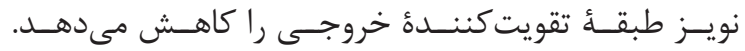

${ }^{1}$ Sinus arrhythmia

${ }^{2}$ Sinus bradycardia

${ }^{3}$ Sinus tachycardia

${ }^{4}$ Electrocardiogram

${ }^{5}$ Holter monitor
مقدمه

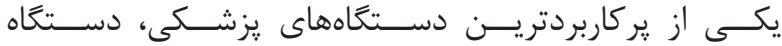

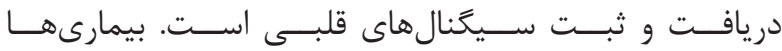

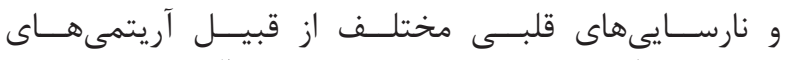

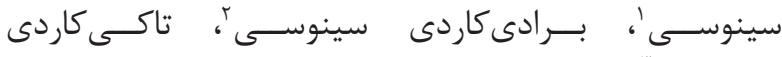

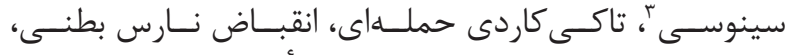

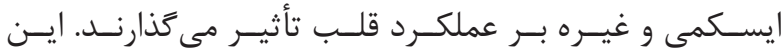

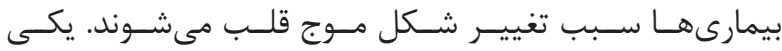

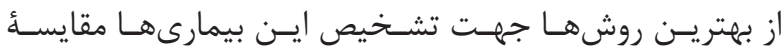

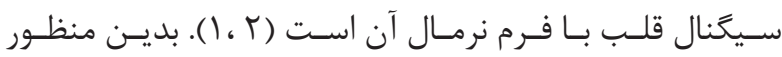

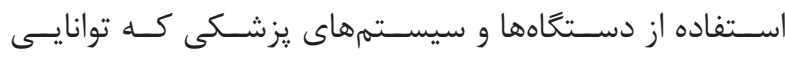

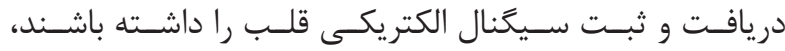

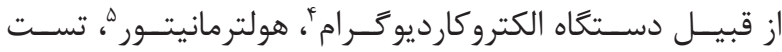

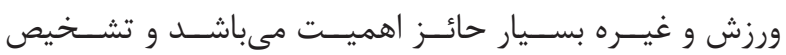

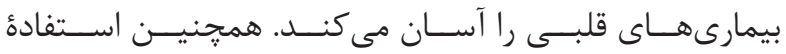

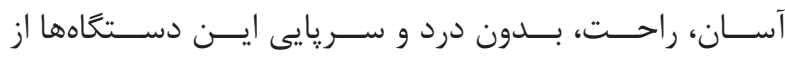

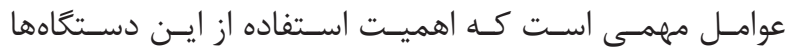

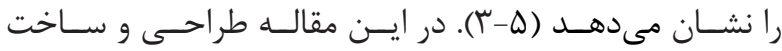

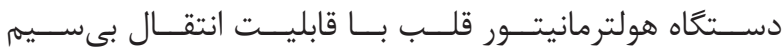

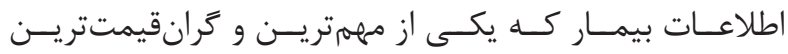

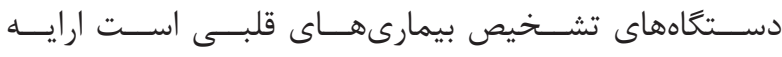

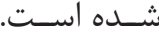

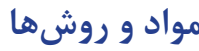

شـماى كلـى سيسـتم ثبـت سـيكَنال قلبـى در نمـودار تصويـرـ

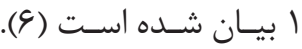
بخش آنالوى

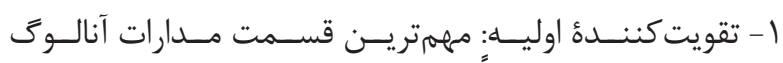

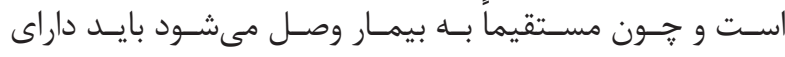

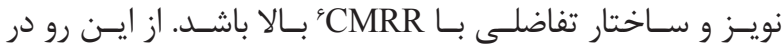

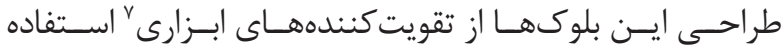

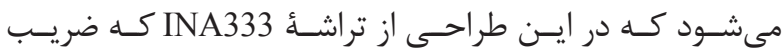

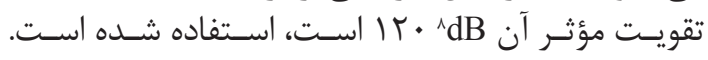

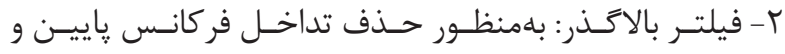

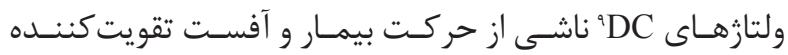

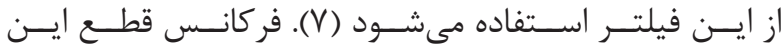

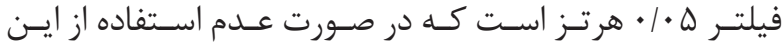

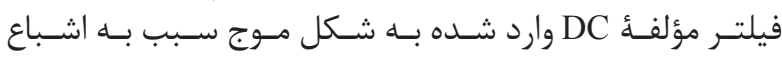

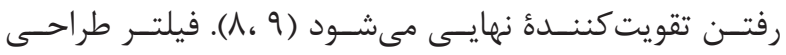

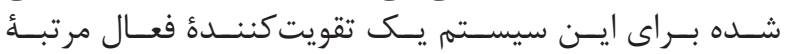

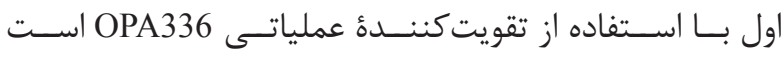

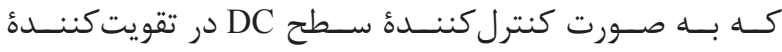

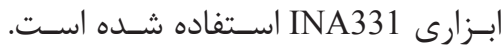

${ }^{6}$ Common-mode rejection ratio

${ }^{7}$ Instrumental amplifier

${ }^{8}$ Decibel

${ }^{9}$ Direct current 
$\ddot{0}$
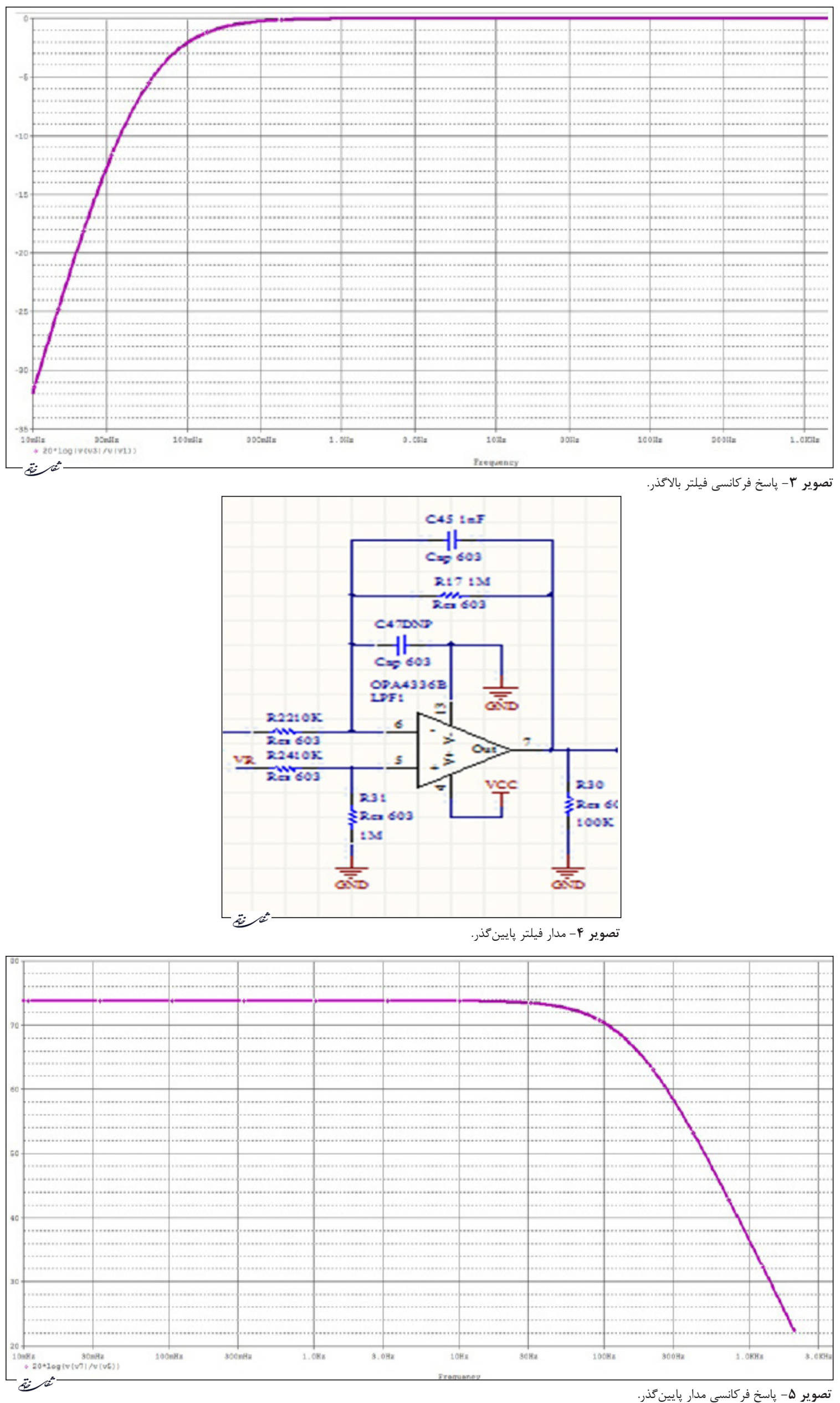


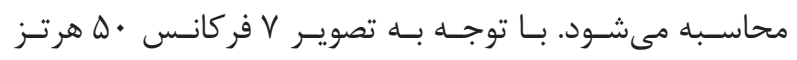

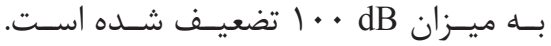

$$
\begin{aligned}
& F=\frac{1}{2 \pi R C} \\
& \text { فر كانس قطع dB }
\end{aligned}
$$

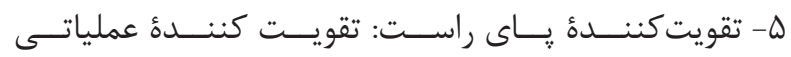

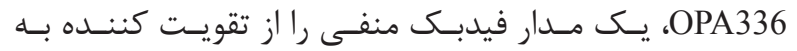

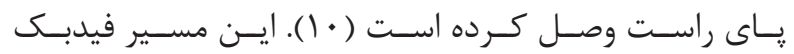

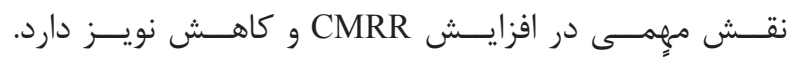

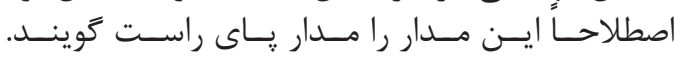

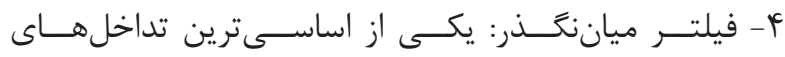

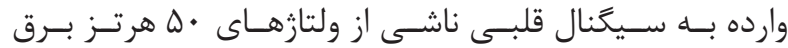

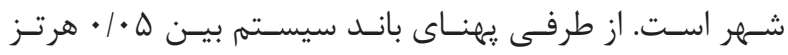

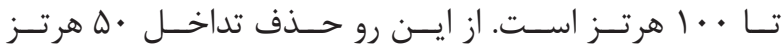

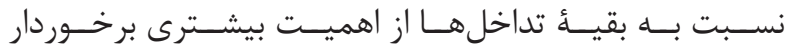

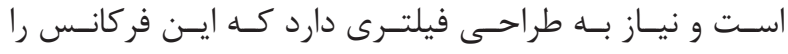

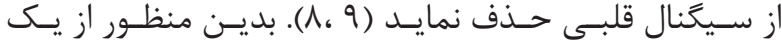

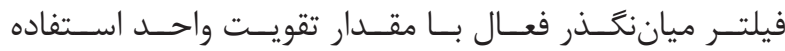

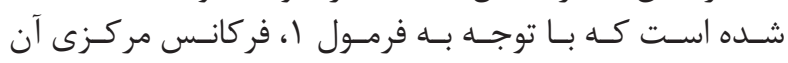
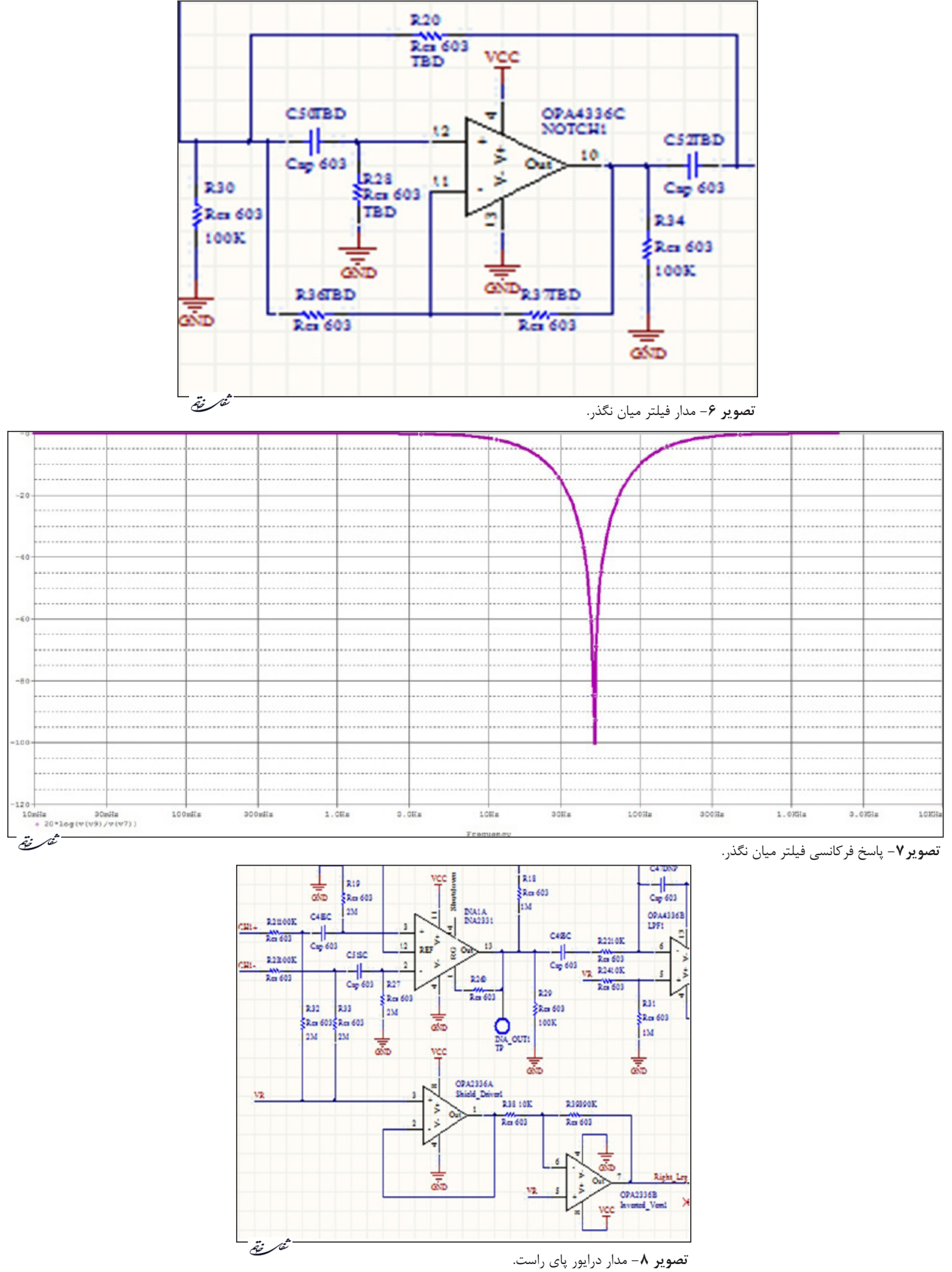


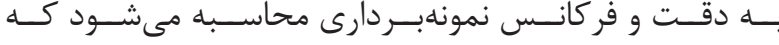

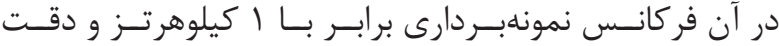

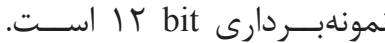

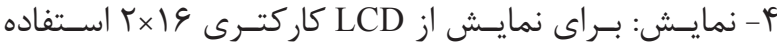

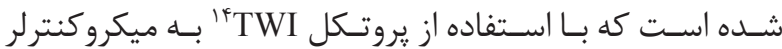

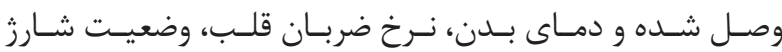

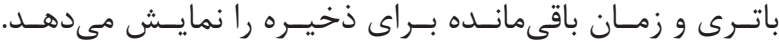
انتقال بىسيم

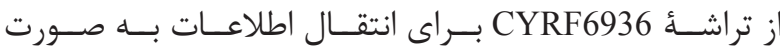

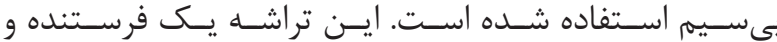

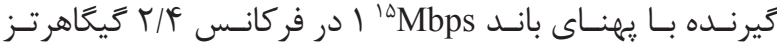

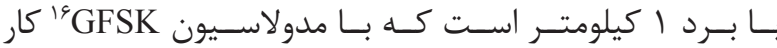

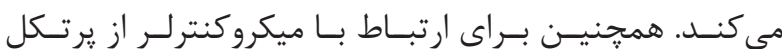

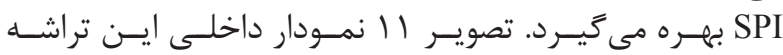

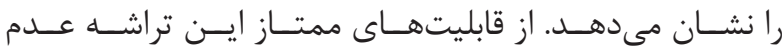

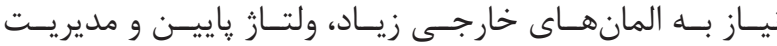

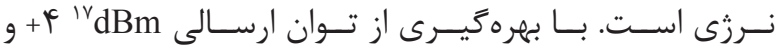

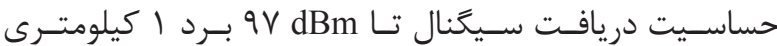

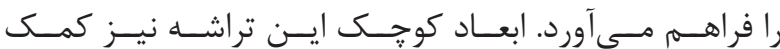

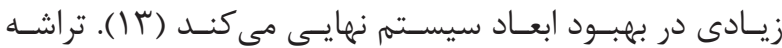

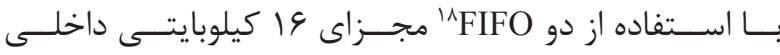

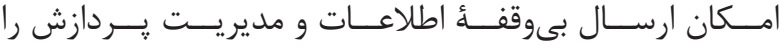

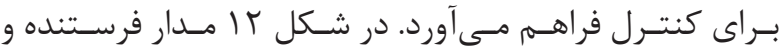

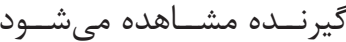

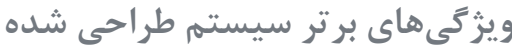

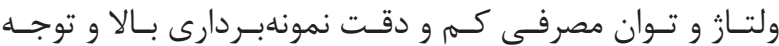

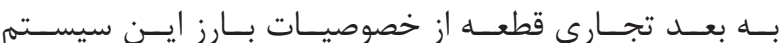

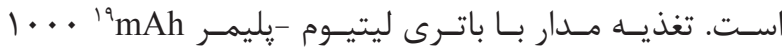

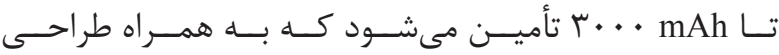

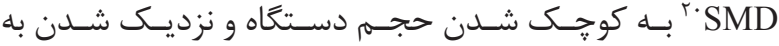

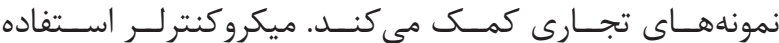

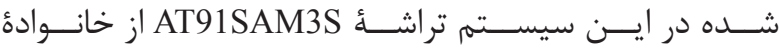

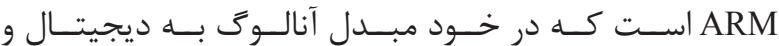

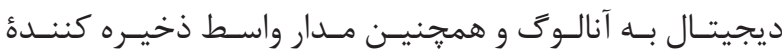

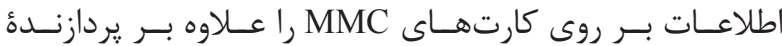

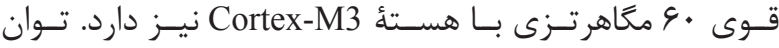

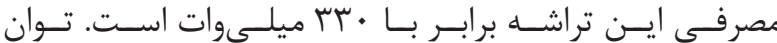

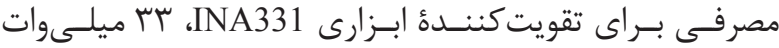

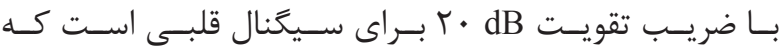

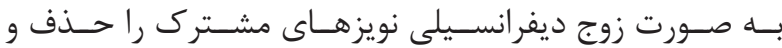

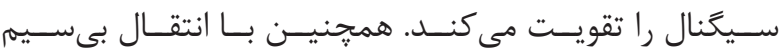

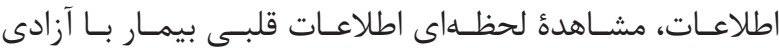

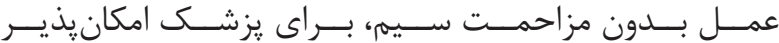

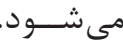

${ }^{10}$ Microcontroller

${ }^{11}$ MMC card

${ }^{12}$ Serial peripheral interface

${ }^{13}$ High-speed multimedia card interface

${ }^{14}$ Two wire interface

${ }^{15}$ Mega bit per second
بخش ديجيتال

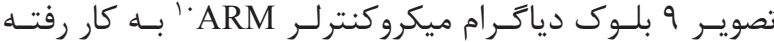

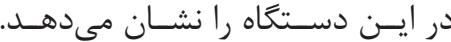

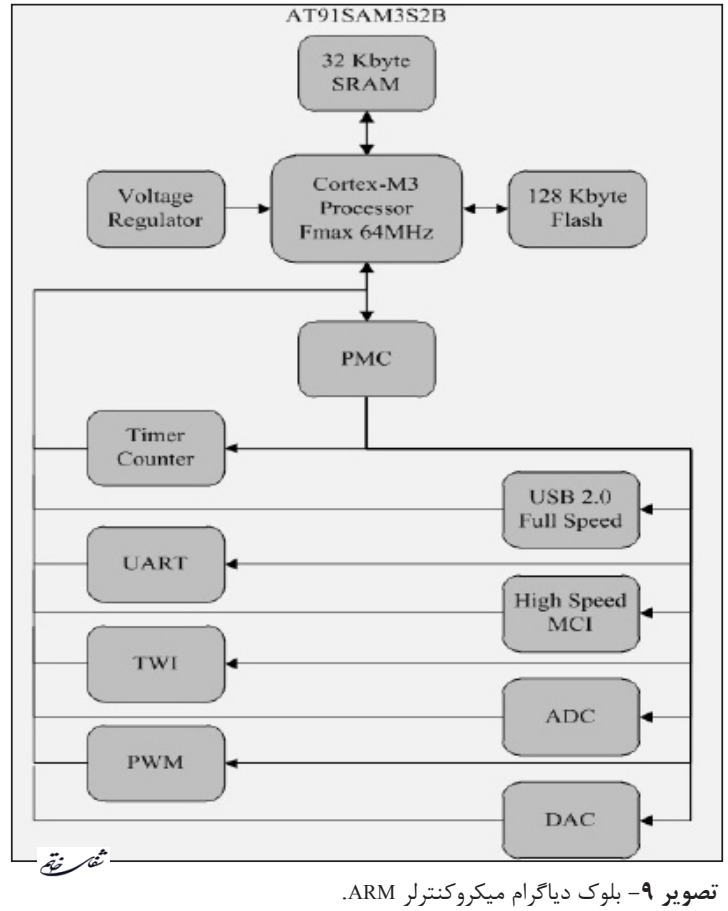

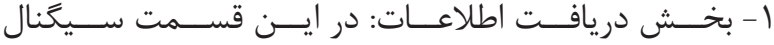

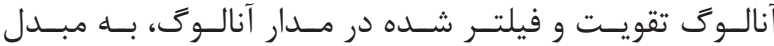

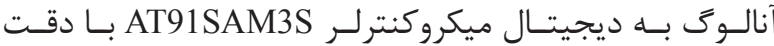

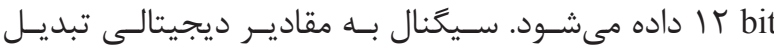

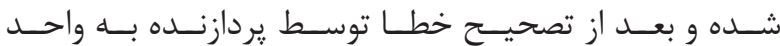

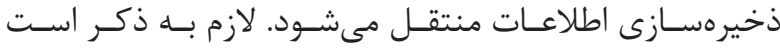

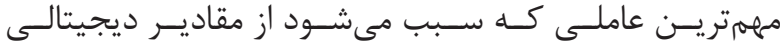

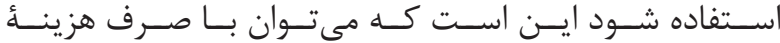

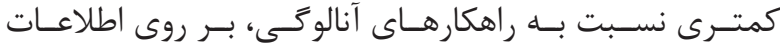

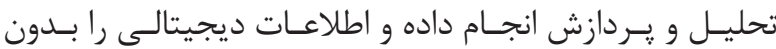

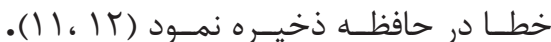

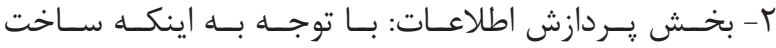

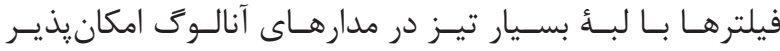

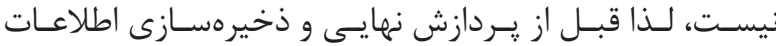

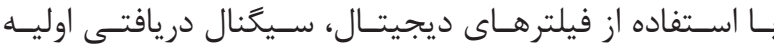

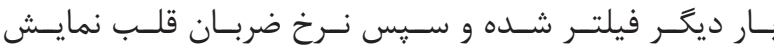
داده مىشـود (1 ار ، 11).

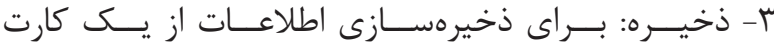
MMC

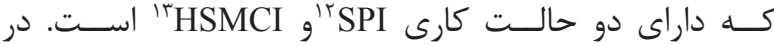

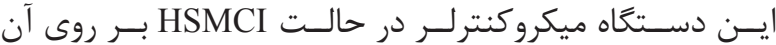

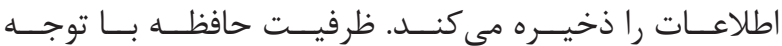

${ }^{16}$ Gaussian frequency shift keying

${ }^{17}$ Decibel millivolts

${ }^{18}$ first in, first out

${ }^{19}$ Mill ampere hour

${ }^{20}$ Surface-mount device 

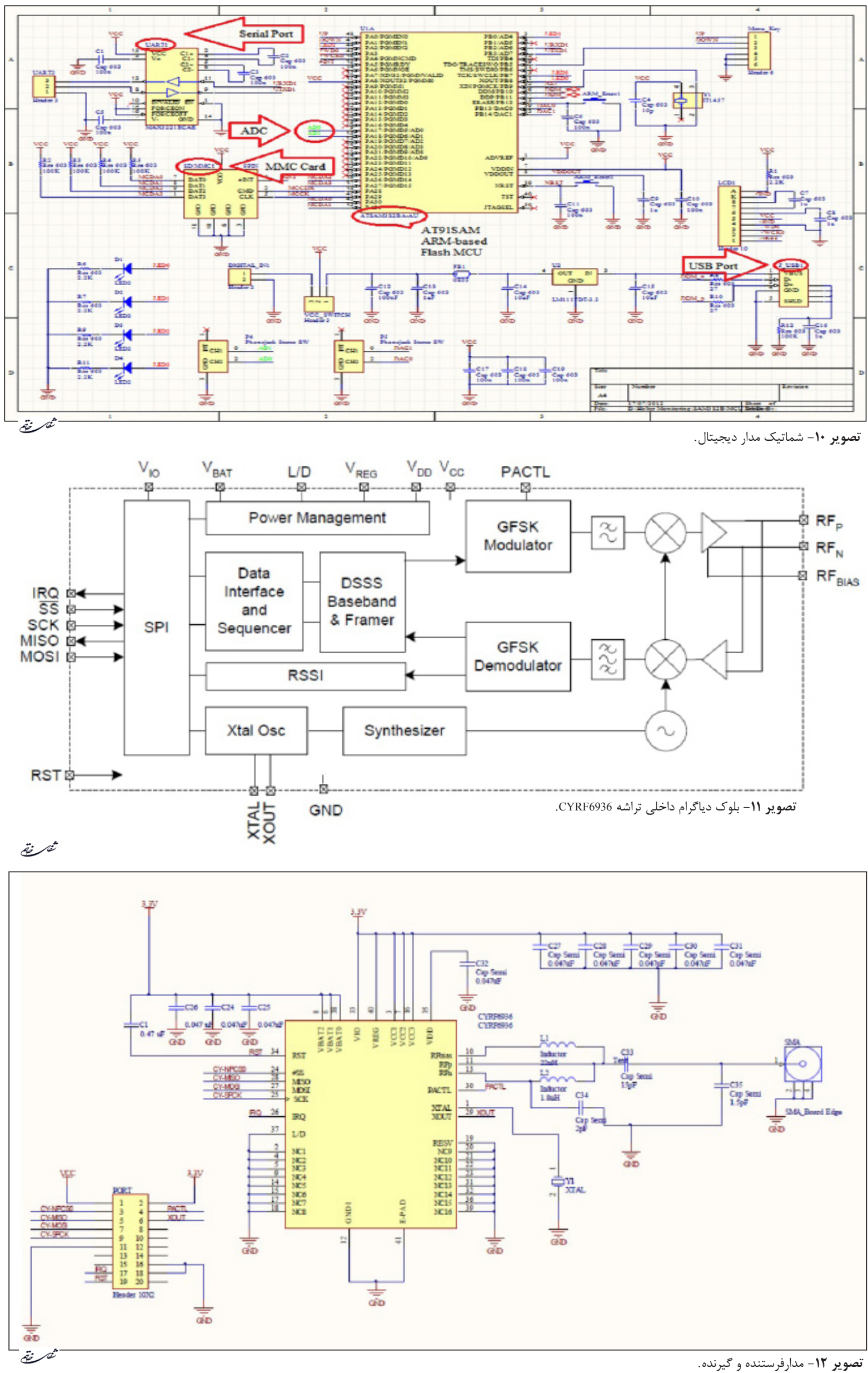


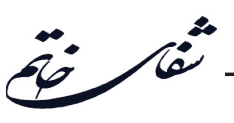

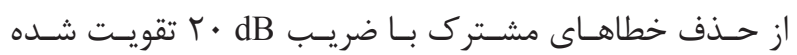

كافته ها

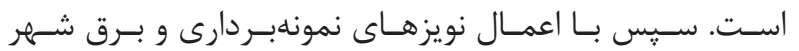

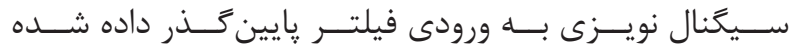

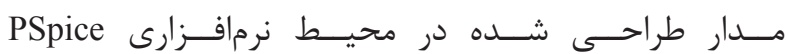

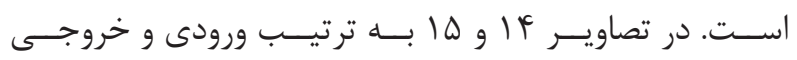

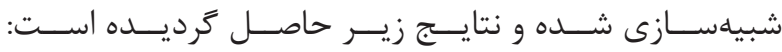

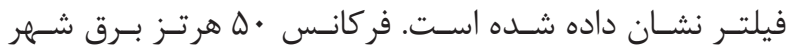

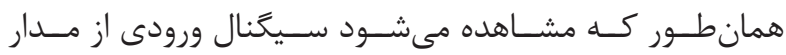

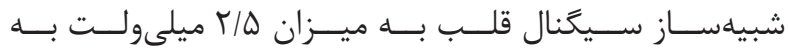

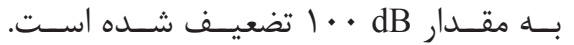

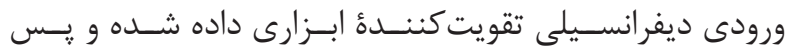
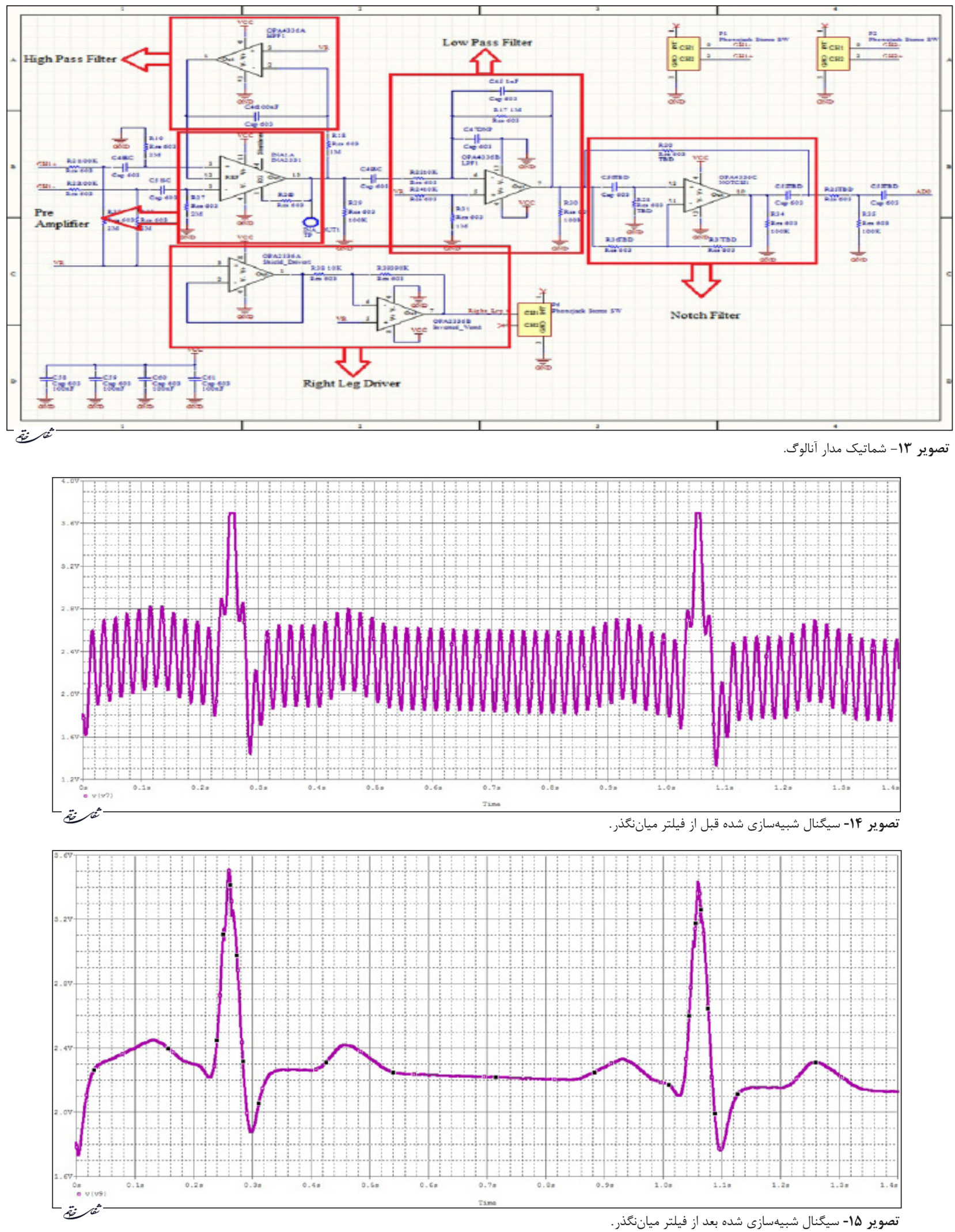


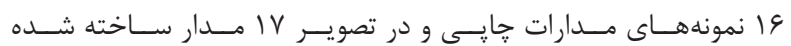

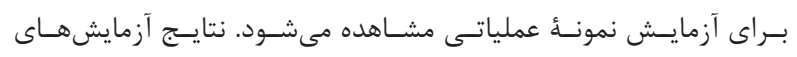

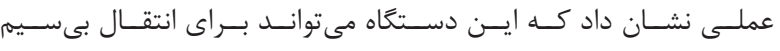

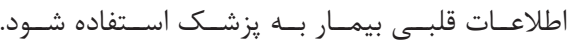

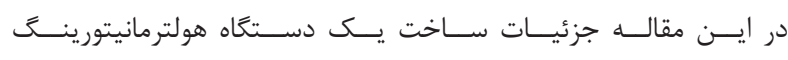

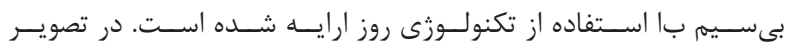
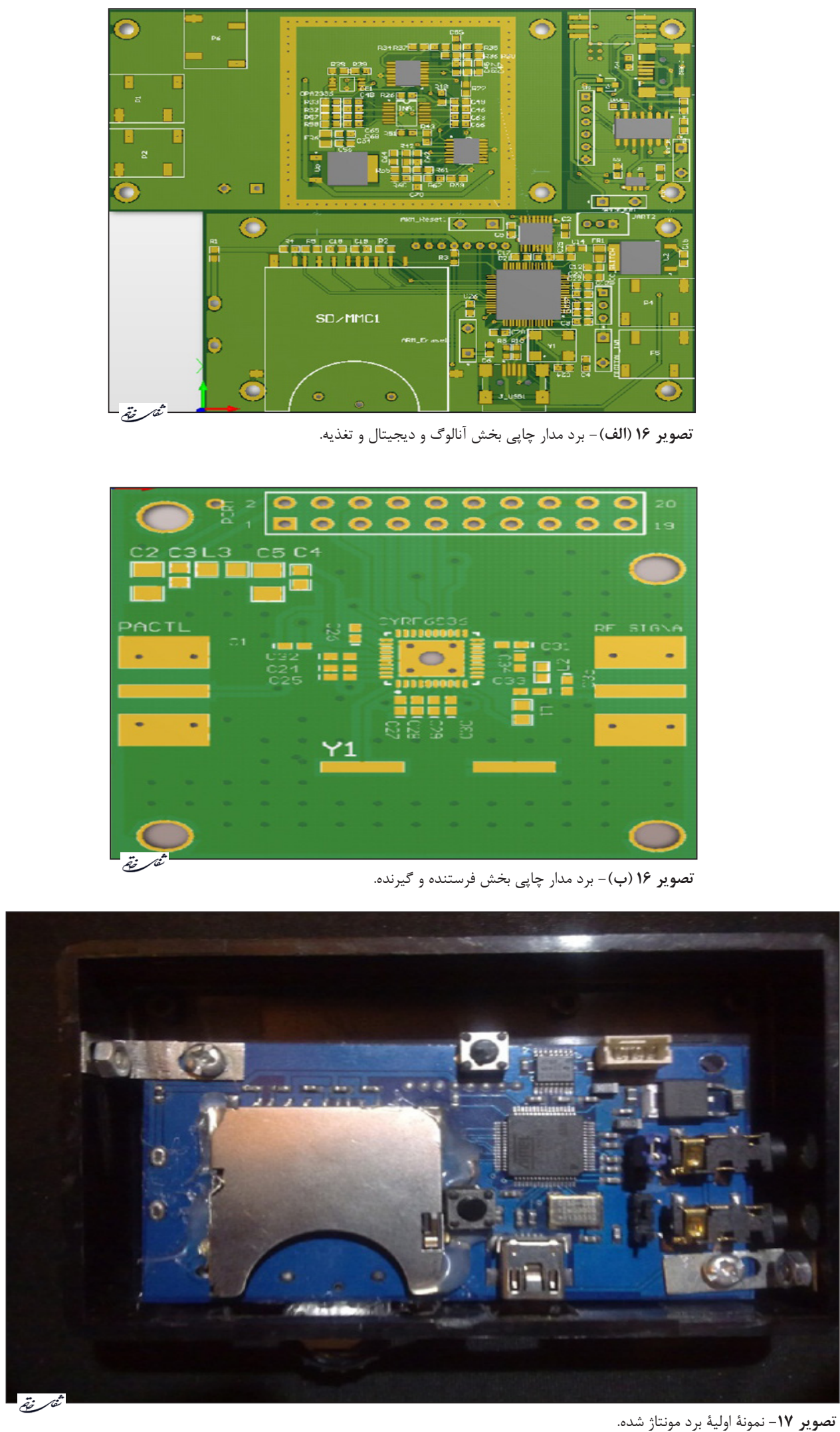
1. Amiri P. Principles of design and construction of Holter monitoring. Msc thesis. K.N Toosi University of Technology. 1997.

2. Jallalian Sh, Sotoodeh B. Electrocardiographs devices. Bsc thesis. Islamic Azad University, South Tehran Branch. 2008.

3. Ho CS, Chiang TK, Lin CH, Lin PY, Cheng JL, Ho $\mathrm{SH}$. Design of portable ECG recorder with USB storage. EDSSC 2007. IEEE Conference. 2007. P. 1095-8. Doi: 10.1109/EDSSC.2007.4450319.

4. Gnecchi JA, Vargas FO, Peregrino VH, Espinoza DL. Design and construction of a continuous ambulatory electrocardiogram recorder, auxiliary in the detection of cardiac arrhythmias. Electronics, Robotics and Automotive Mechanics Conference IEEE. 2010. p. 602-6.

5. Hornos T. Wireless ECG/EEG with the MSP430 microcontroller. Msc thesis. Glasgow University. 2009.

6. Shahrokhi MJ, Hajazim S. Design, simulation and implementation of heart Holter monitoring. Bsc thesis. Shaid Rajaee Teacher Training University. 2012.

\section{منابع}

7. Wireless ECG Monitoring System. http://www.frbiz. com/j34375533-wireless-ecg-monitoring-system.html.

8.MohammadZadehF.Contactlessmechanocardiograph. Msc thesis. Simon Fraser University. 2008.

9. Jin H, Miao B. Design of holter ECG system based on MSP430 and USB technology. In2007 1st International Conference on Bioinformatics and Biomedical Engineering. (ICBBE), p. 976-979.

10. Vavrinsky E, Daricek M, Moskalova D, Horinek F, Donoval M. Design of very precise and miniature low power ECG holter. Electrocardiology 2014: Proceedings of the $41 \mathrm{st}$ International Congress on Electrocardiology.

11. Diagnostic ECG. http://www3.gehealthcare.com/en/ products/categories/diagnostic_ecg.

12. Analog Device Inc. http://users.ece.utexas. edu/ valvano/Datasheets/AD620.pdf.

13. Forest medical introduces trillium 5000/gold holter monitoring system. http://www.forestmedical.com/ pr04.htm. 